University of Nebraska - Lincoln

DigitalCommons@University of Nebraska - Lincoln

Publications from USDA-ARS / UNL Faculty

U.S. Department of Agriculture: Agricultural

Research Service, Lincoln, Nebraska

7-30-2008

\title{
Human dispersal of Trichinella spiralis in domesticated pigs
}

\author{
Benjamin Rosenthal \\ United States Department of Agriculture, Benjamin.Rosenthal@ars.usda.gov \\ Giuseppe LaRosa \\ Istituto Superiore di Sanita \\ Dante Zarlenga \\ United States Department of Agriculture \\ Detiger Dunams \\ United States Department of Agriculture \\ Yao Chunyu \\ Jilin University
}

See next page for additional authors

Follow this and additional works at: https://digitalcommons.unl.edu/usdaarsfacpub

Rosenthal, Benjamin; LaRosa, Giuseppe; Zarlenga, Dante; Dunams, Detiger; Chunyu, Yao; Mingyuan, Liu; and Pozio, Edoardo, "Human dispersal of Trichinella spiralis in domesticated pigs" (2008). Publications from USDA-ARS / UNL Faculty. 2255.

https://digitalcommons.unl.edu/usdaarsfacpub/2255

This Article is brought to you for free and open access by the U.S. Department of Agriculture: Agricultural Research Service, Lincoln, Nebraska at DigitalCommons@University of Nebraska - Lincoln. It has been accepted for inclusion in Publications from USDA-ARS / UNL Faculty by an authorized administrator of DigitalCommons@University of Nebraska - Lincoln. 


\section{Authors}

Benjamin Rosenthal, Giuseppe LaRosa, Dante Zarlenga, Detiger Dunams, Yao Chunyu, Liu Mingyuan, and Edoardo Pozio 


\title{
Human dispersal of Trichinella spiralis in domesticated pigs
}

\author{
Benjamin M. Rosenthal ${ }^{\mathrm{a}, *}$, Giuseppe LaRosa ${ }^{\mathrm{b}}$, Dante Zarlenga ${ }^{\mathrm{a}}$, Detiger Dunams ${ }^{\mathrm{a}}$, \\ Yao Chunyu ${ }^{c}$, Liu Mingyuan ${ }^{c}$, Edoardo Pozio ${ }^{b}$ \\ a Animal Parastic Diseases Laboratory, Agricultural Research Service, United States Department of Agriculture, Building 1180, Beltsville, MD 20705, USA \\ ${ }^{\mathrm{b}}$ Department of Infectious, Parasitic and Immunomediated Diseases, Istituto Superiore di Sanità, viale Regina Elena, 299-00161 Rome, Italy \\ ${ }^{\mathrm{c}}$ Key Laboratory of Zoonoses, Ministry of Education, Institute of Zoonoses, Jilin University, 5333 Xian Road, 130062 Changchun, PR China
}

\section{A R T I C L E I N F O}

\section{Article history:}

Received 5 June 2008

Received in revised form 18 July 2008

Accepted 19 July 2008

Available online 30 July 2008

\section{Keywords:}

Trichinella

Swine

Domestication

Epidemiology

Population genetics

Evolution

Population

Structure

Pigs

Parasite

Zoonosis

Zoonoses

Public health

Agriculture

\begin{abstract}
A B S T R A C T
To investigate the human impact on the evolutionary ecology of animal pathogens, we compared genetic diversity of severe foodborne parasites contracted by eating infected pork or wild game. In particular, we characterized Trichinella spp. from twenty-eight countries and four continents by genotyping nine microsatellite loci and sequencing one-fifth of the mitochondrial genome. All specimens of Trichinella spiralis, a swine parasite that can infect many species of wildlife, were remarkably uniform across Europe, North Africa, and the Americas. Far greater diversity characterized a comparable sample of Trichinella britovi, which parasitizes various sylvatic mammals endemic to Eurasia and North-Western Africa. A limited sample of $T$. spiralis in Asia, where swine were first domesticated, encompassed greater genetic variability than those in the West, as did small samples of Trichinella nativa and Trichinella murrelli, which parasitize wildlife hosts. We conclude that European lineages of $T$. spiralis originated several thousand years ago, approximately when pigs were first domesticated there. These data also imply that Europeans inadvertently introduced $T$. spiralis to the Americas via infected pigs and/or rats. Despite evidence that early hominid hunters ingested foodborne parasites by hunting wild game millions of years earlier, swine husbandry has governed the subsequent transmission, dissemination, and evolutionary diversification of T. spiralis. Where viable parasites have been eliminated from their diet, the residual risk posed to swine by exposure to wildlife or rats should be more precisely defined because breaking the cycle of transmission would confer enduring economic and health benefits.
\end{abstract}

Published by Elsevier B.V.

\section{Introduction}

Humans may be "the world's greatest evolutionary force" (Palumbi, 2001), inducing long-lasting change in other species. Animals and plants first domesticated $\sim 9000$ years ago still define our diet and landscape (Clutton-Brock, 1999; Diamond, 2002). Although early hominids first contracted certain animal parasites millions of years ago (Hoberg et al., 2001), efficient human transmission of other animal-derived pathogens was a consequence of intensive husbandry of animals. Diseases derived from Eurasian livestock, such as smallpox and measles, facilitated European colonial expansion by devastating previously unexposed human populations (Crosby, 1976, 1986). As present concerns over the pandemic potential of Avian Influenza attest, public health continues to be threatened when intensive agriculture exposes

\footnotetext{
* Corresponding author. Tel.: +1 301504 5408; fax: +1 3015048979.

E-mail address: benjamin.rosenthal@ars.usda.gov (B.M. Rosenthal).
}

people to communicable animal pathogens (Horimoto and Kawaoka, 2005). Have humans engendered evolutionary change only in those pathogens transmitted from person to person, or also in those we contract from other animals? Herein, we examined whether or not domesticating swine helped establish and disseminate Trichinella spiralis, a highly pathogenic foodborne parasite (Dupouy-Camet et al., 2002).

To investigate the human impact on the evolutionary ecology of animal pathogens, we compared the genetic diversity of $T$. spiralis obtained from wildlife and domesticated pigs. To this end, we compared the diversity of parasite populations in Asia, where pigs were first domesticated in the Neolithic from several lineages of wild boar, and in Europe, where pigs were subsequently domesticated from distinct, genetically limited ancestors (Larson et al., 2005, 2007). In particular, we sought to understand whether the older and more genetically diverse pigs of Asia might harbor correspondingly more diverse parasite populations.

Nematodes in the genus Trichinella are acquired by ingesting uncooked meat (striated muscle) in which larval parasites have 
Table 1

Specimen origins and genotypes

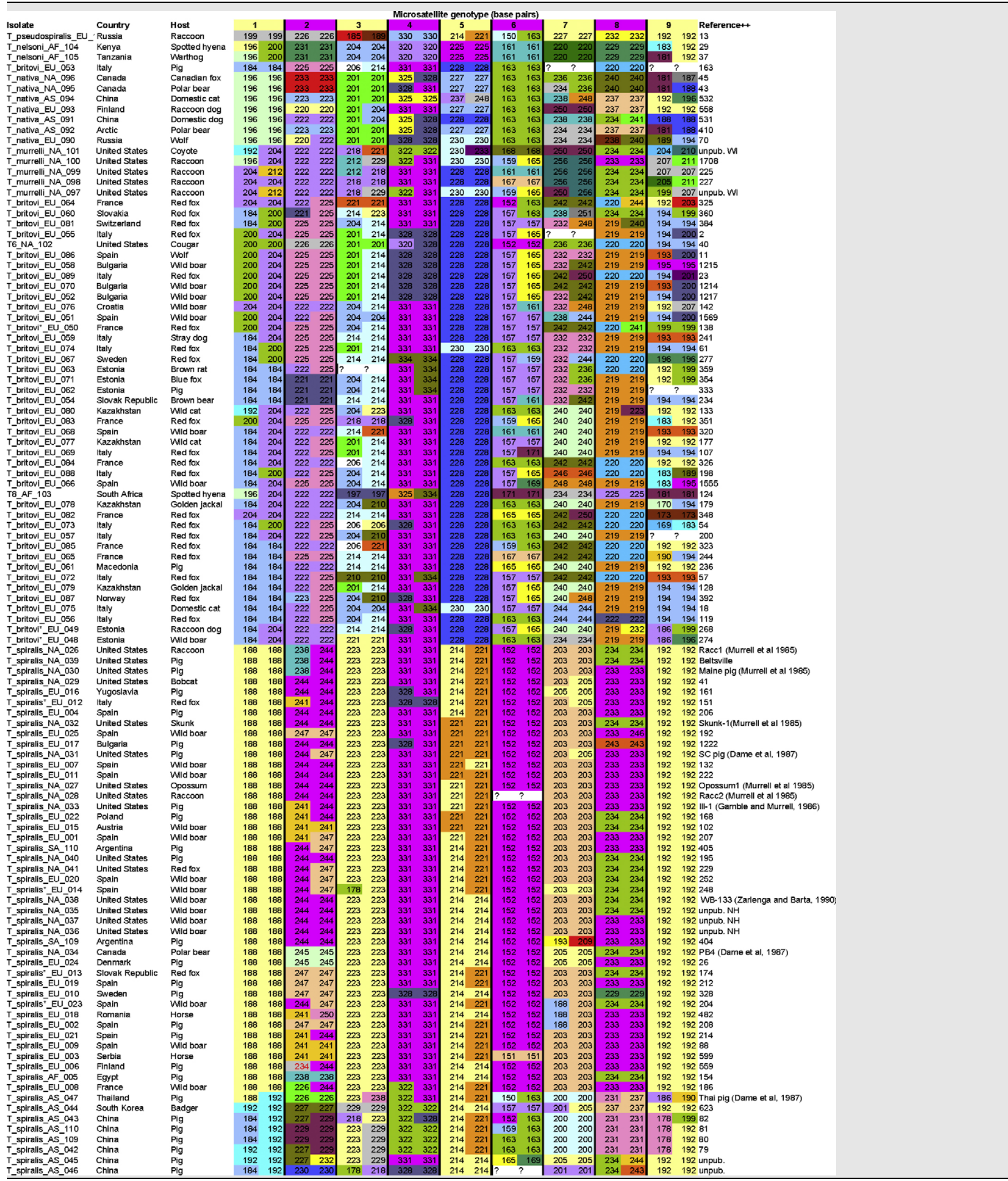

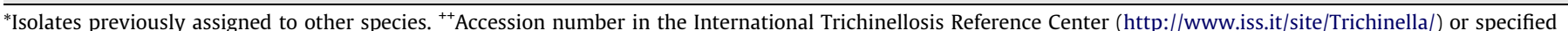
publication. See Refs. (Dame et al., 1987; Gamble and Murrell, 1986; Murrell et al., 1985; Zarlenga and Barta, 1990). 
encysted. Most species of Trichinella occupy well-defined geographic ranges (Zarlenga et al., 2006). Trichinella britovi parasitizes various sylvatic mammals (canidae, felidae, mustilidae, ursidae, viverridae and suidae) endemic to Eurasia and North-Western Africa, whereas Trichinella murrelli is restricted to North American wildlife (Kapel, 2000; Pozio and Murrell, 2006). Domesticated swine may become infected with $T$. spiralis if they are permitted to eat uncooked meat (Pozio and Murrell, 2006). Although swine represent the most significant source of human exposure to $T$. spiralis, this parasite is capable of infecting a wide range of other mammals (Kapel, 2000; Pozio, 2000; Pozio and Murrell, 2006).

To compare the extent and pattern of genetic variability in ecologically distinct species of Trichinella, we defined the variability of nine nuclear microsatellite loci and sequenced approximately one-fifth of the mitochondrial genome from the broadest population sample yet attempted for species of Trichinella, involving twenty-eight countries on four continents. We discovered that $T$. spiralis in domesticated pigs throughout the Western world share strikingly uniform genotypes. By implicating permissive agricultural practices as responsible for the parasite's historical dissemination, these data motivate renewed interest in understanding whether such practices remain necessary for this parasite's continued transmission.

\section{Methods}

\subsection{Specimens}

Decades of sampling contributed to our broad survey of specimens, most of which were derived from the International Trichinella Reference Centre in Rome, Italy (http://www.iss.it/site/ Trichinella). Each specimen and its origin is specified in Table 1. Our study emphasized a comparison between $T$. spiralis and $T$. britovi, including additional specimens of other available species and genotypes for added context.

\subsection{DNA extraction}

DNA was extracted from small pools $(\sim 15)$ of larvae that were isolated from fresh tissue or from long-term liquid nitrogen storage, using Proteinase K digestion and adsorption to magnetic beads using the DNA-IQ system and the Tissue and Hair extraction kit (Promega).

\subsection{PCR amplification}

The primers for each locus, and the repeat motif they flank, are specified in Table 2 . To identify microsatellite loci, we screened the draft $T$. spiralis genome project in silico for simple repeat motifs. Candidate loci were screened via PCR for their ability to amplify robust products under a range of annealing temperatures. We
Table 3

Mitochondrial sequencing primers

\begin{tabular}{ll}
\hline Forward & Reverse \\
\hline CACATGATTCACAATCACCT & GAAGCTTAAAATGTCTTCTC \\
GCAGTAAGAAACCCATCAGA & TAAGTAAGATTTCAATGGCG \\
GGAGTAACCAAAAATCTAGATCCAA & AAATCTTAAGTACTCGTAGTTTA \\
CTAGAATGAAAGGAGCAAAG & AGGTTGTGATTATTAGTTTCTAGGG \\
CCACAATTACCTTACTAATCAC & CCACAATTACCTTACTAATCAC \\
ACACACCATTAGGATGAATA & AGGAATACACCTACGATTAA \\
GCATGTCTAAGACTAATTGCATCA & CCTAGTCAGGAGGAGTTGGG
\end{tabular}

confirmed the target locus using agarose gel electrophoresis and bi-directional fluorescent sequencing using BigDye v.3.1 chemistries. Thereafter, microsatellite genotyping was accomplished via capillary electrophoresis of products labelled with 6-FAM on an ABI 3730 DNA sequencer. Alleles were called using Genemapper software (Applied Biosystems).

Approximately $3100 \mathrm{bp}$ of contiguous mitochondrial DNA (spanning cytb, tRNA-Ser, SSU rDNA, tRNA-Val, LSU rDNA, atp6, and cox3 genes) were sequenced from each of 14 Western and 8 Asian isolates of T. spiralis. Primers Trichi-cob-F1 and Trichi-cox3R1 (Lavrov and Brown, 2001) were used in conjunction with a series of internal primers designed from a full-length mitochondrial genome sequence AF293969 (Table 3).

Lyophilized DNAs were reconstituted in $50 \mu \mathrm{l}$ water prior to use. Polymerase chain reactions $(20 \mu \mathrm{l})$ were comprised of $2 \mu \mathrm{l}$ DNA, $0.2 \mathrm{mM}$ dNTPs, $0.5 \mu \mathrm{M}$ of each primer, and either $0.5 \mathrm{U}$ of Platinum ${ }^{\circledR}$ High Fidelity Taq polymerase (Invitrogen) in $0.6 \mathrm{mM}$ $\mathrm{MgSO}_{4} 1 \times$ buffer (Invitrogen), or $0.5 \mathrm{U}$ Native Taq polymerase (Fisher Scientific) in $0.6 \mathrm{mM} \mathrm{MgCl} 21 \times$ PCR buffer. Negative control reactions were included in each experiment. Each PCR commenced with 3 min denaturation at $94{ }^{\circ} \mathrm{C}$ and culminated with a final 10 min extension at $72^{\circ}$. Microsatellite loci were amplified using $35-40$ cycles @ $94^{\circ}$ for $30 \mathrm{~s}, 53^{\circ}$ for $45 \mathrm{~s}$, and $72^{\circ}$ for $90 \mathrm{~s}$, using 6FAM labelled forward primers; mitochondrial loci were amplified using 35 cycles @ $94^{\circ}$ for $30 \mathrm{~s}, 55^{\circ}$ for $30 \mathrm{~s}$, and $72^{\circ}$ for $2 \mathrm{~min}$.

\subsection{Microsatellite length determination}

Amplified products were diluted 50-fold in water and mixed $1: 10$ in $\mathrm{Hi}-\mathrm{Di}^{\mathrm{TM}}$ Formamide containing $0.75 \%$ GeneScan $^{\mathrm{TM}} 500 \mathrm{LIZ}^{\mathbb{R}}$ Size Standard. Samples were electrophoresed on an Applied Biosystems 3730 DNA Analyzer and genotyped using Genemapper $^{\circledR}$ v.3.7.

\subsection{Sequencing}

To prepare PCR products for dual-directional sequencing, excess primers and dNTPs were removed by adding $0.8 \mu$ l of ExoSap-IT ${ }^{\mathbb{R}}$ (USB Corp.) to $2 \mu \mathrm{l}$ of the PCR product. After successive $15 \mathrm{~min}$ incubations at $37^{\circ}$ and $80^{\circ}$, sequencing reactions were completed by adding $1 \mu \mathrm{l}$ of BigDye ${ }^{\circledR}$ Terminator v.3.1, $2 \mu$ l of $5 \times$ Big Dye ${ }^{\circledR}$

Table 2

Microsatellite primers and repeat motifs

\begin{tabular}{|c|c|c|c|c|}
\hline Locus & Alias & Forward & Reverse & Repeat \\
\hline 1 & TP1 & GCGCGATTACGACACTACAA & ATTCGCCACTGTCACTTTCC & TTAA \\
\hline 2 & TP5 & TACATGGCCCACAGCAAAT & GATGGCCACCAGGTAAGAAA & TTA \\
\hline 3 & TP19 & AGGAAGATCAAGCGGCAATA & CACGAGTTTGCCTGATGAAA & CAA \\
\hline 4 & TP26 & GACGTTCAAGAAACGAATGCT & GGATAACCCTCGGCGTATTT & AAC \\
\hline 5 & TP28 & TCGTTTTTCGTGCTTGATTG & CGGACTTGGTTGCTAGTTGA & TTAAAA \\
\hline 6 & TP32 & GCGGGTGAAAAATTTCTCTTT & TCAGTCGAAGCAAACCAAAA & TG \\
\hline 7 & TP43 & TACAGGCGTTCGACACAATC & AGCGCTGAGGTGTCTTTCAT & TA \\
\hline 8 & TP47 & GAACAGCTTCGGTAGGATGC & TGAATGGCGTGTTTGACAAT & TA \\
\hline 9 & TP53 & TTGCACAAGTGCGAAAACTC & TGGGTGTGATAGCAACCAGT & TG \\
\hline
\end{tabular}


terminator buffer (Applied Biosystems) and $1 \mu \mathrm{l} 3.2$ pmol primer. These underwent 25 cycles @ $92^{\circ}$ for $15 \mathrm{~s}, 50^{\circ}$ for $15 \mathrm{~s}, 60^{\circ}$ for $4 \mathrm{~min}$. Unincorporated fluorescent dNTPs were then removed using gel cartridge columns (Edge Biosystems) prior to electrophoresis on an ABI 3100 sequencer. Sequence chromatograms were edited using Sequencher ${ }^{\circledR}$ V.4.6 (Genecodes Corp.). Sequence data were aligned in Vector NTI Advance v.10 (Invitrogen Corp.).

Mitochondrial gene trees were reconstructed under the criterion of minimum evolution from Kimura 2-parameter distances using MEGA 4.2 (Tamura et al., 2007).

\subsection{Genotype-based assignment of individuals to populations}

Structure 2.2 was used to assess the statistical confidence with which each specimen could be assigned to one (or more) population subdivisions. Each of ten replicate analyses sampled 1 million generations after discarding a 'burn-in' period of 100,000 generations, under the assumption of seven populations. The statistical plausibility and population composition of alternative scenarios, assuming less or more population subdivision, was also evaluated. These resulted in qualitatively similar outcomes: whereas parasites of wildlife hosts could be further subdivided, Western isolates of $T$. spiralis could not (data not shown).

\subsection{Divergence time estimation}

The mean difference in microsatellite length, $(\partial \mu)^{2}$ provides an accurate estimate of divergence time irrespective of changes in population size when this estimator has not yet asymptotically approached its maximum value (Goldstein et al., 1995). Its application to the divergence between Western and Asian populations of $T$. spiralis appears justified, since its value (40.59) is less than one-sixth the value as when Western T. spiralis and $T$. britovi are compared (257.57).

\subsection{Estimating parasite generation length}

Indirect estimates of parasite longevity were derived by considering the life history of their typical hosts. Traditionally, piglets were weaned at 2-3 months of age, at which point they could first acquire infection. Subsequent transmission could commence soon thereafter (when many such animals were slaughtered) or within 2 or 3 years (their typical maximum age) (Mason, 1986). Parasite longevity would be further reduced in swine dying before their intended slaughter, in swine whose immunity allowed them to outlive their parasites, and in synanthropic rats where transmission would cycle more rapidly.

\section{Results}

One group of $T$. spiralis isolates share remarkably uniform genotypes defined by nine autosomal microsatellite markers (Fig. 1A). This "Western" group, which included every isolate of $T$. spiralis sampled from Europe, the Americas, and Egypt $(n=43)$, harbored fewer alleles and multilocus genotypes than either $T$. britovi from European wildlife $(n=44)$ or $T$. spiralis from Asia

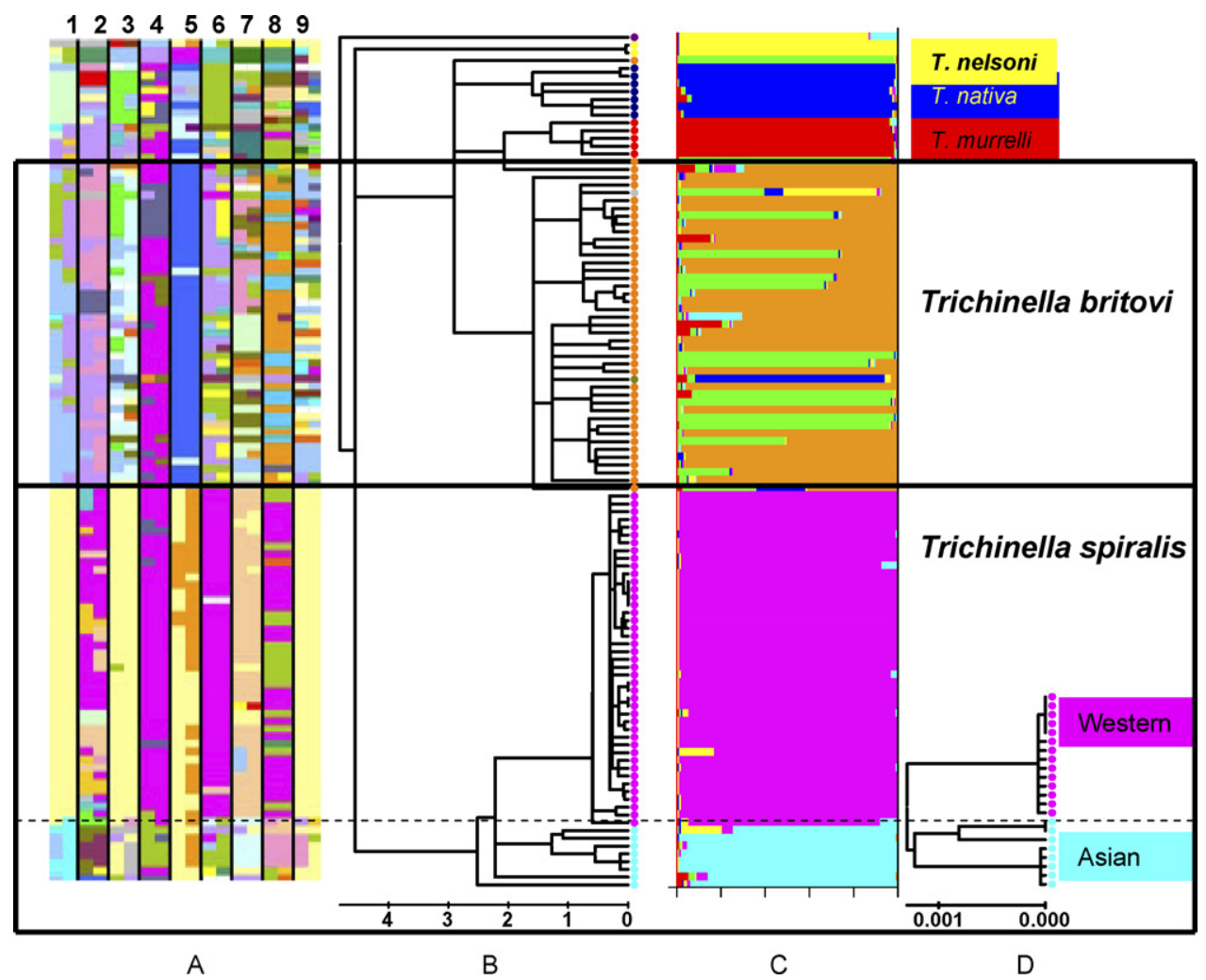

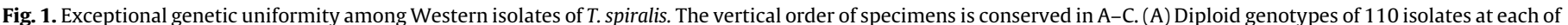

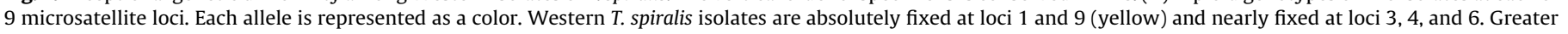

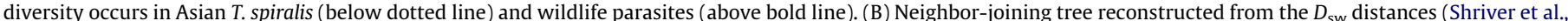

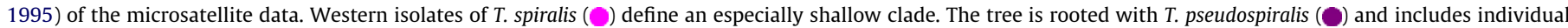

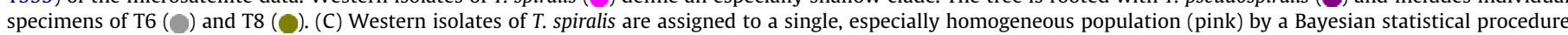

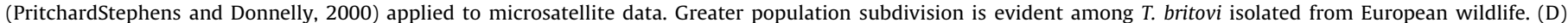

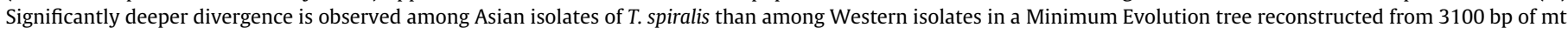
DNA. 
$(n=8)$. Although more than five times as many "Western" than Asian isolates were genotyped, Western isolates harbored more alleles at only one of nine microsatellite loci. The most frequent allele never exceeded 67\% in Asian isolates, but did so in six of nine microsatellite loci in Western isolates. At eight of nine loci, the predominant allele in Western isolates was more frequent than the predominant allele in Asia ( $p<.005$, Wilcoxan signed-rank test). Among polymorphic loci, half as many alleles occur in $T$. spiralis as in $T$. britovi. Despite their derivation from four continents and several host species (including domestic pigs wild boars, red foxes, opossums, raccoons, and horses), the isolates of the Western group in our entire sample set are genetically less variable than those European specimens of $T$. britovi obtained from a single wildlife species (red foxes, $n=14$ ). They are also less variable than our modest sample of either T. murrelli or T. nativa, parasites endemic to wildlife hosts of temperate North America and the Arctic, respectively.

A tree reconstructed from inter-individual differences in microsatellite length confirms that all isolates of $T$. spiralis are monophyletic and that Western isolates share an especially recent common origin (Fig. 1B). Far greater microsatellite divergences are evident among isolates of T. spiralis in Asia, and among isolates of $T$. britovi and other taxa of Trichinella in wildlife. These data reaffirm the earlier conclusion (Zarlenga et al., 2006) that $T$. spiralis and a species endemic to African carnivores, Trichinella nelsoni, diverged prior to the lineage of Holarctic species comprised of $T$. britovi, Trichinella nativa, and T. murrelli, but point to a recent dissemination of $T$. spiralis in the West.

Using a Bayesian statistical procedure (Pritchard et al., 2000) that assigns individuals to subpopulations based solely on their microsatellite genotypes without reference to a priori taxonomic or geographic designations, each Western $T$. spiralis isolate was unambiguously assigned to one population, whereas Asian isolates of $T$. spiralis and isolates of $T$. britovi were assigned to other, more variable population subdivisions (Fig. 1C). Comparatively deeper branching in clades defined by T. nativa and $T$. murrelli again attests to their apparently greater degree of genetic variability.

Assuming that each microsatellite locus sustained an average of $5.6 \times 10^{-4}$ mutations (Goldstein et al., 1995) during parasite generations averaging six months, Western and Asian lineages of T. spiralis would have diverged $\sim 18,000$ years ago. Greater precision in this estimate would be possible if the mutation and transmission rates were better known. To further test whether the geographically widespread Western isolates of $T$. spiralis share an especially recent common ancestry, we characterized the extent of variation evident in a substantial portion of the maternally inherited mitochondrial genome. Each specimen of Western T. spiralis had one of only two haplotypes differing at only one of 3100 base pairs ( $d=0.00032$ ). By contrast, among only eight sampled Asian isolates, three mitochondrial haplotypes were identified that differed by as much as eight times that amount (Fig. 1D; Fig. 2). If mitochondrial lineage pairs accumulated differences at a roughly constant rate of $\sim 2 \%$ per million years (Brown et al., 1979), Western T. spiralis matrilineages would have undergone a population bottleneck within the last 16,000 years. That bottleneck might have occurred only 6000 years ago if mitochondrial substitutions actually accumulated 2.6 times faster, as implied by models that account for substantial variation in the substitution rate among sites in mitochondrial genes (Arbogast et al., 2002).

\section{Discussion}

Because the evolutionary ecology of Trichinella spp. has been insufficiently examined (Tibayrenc, 2001), we undertook a global survey of population genetic variation in parasites that exploit wildlife hosts and domesticated swine. Developing a suite of markers which show promise in elucidating the history and diversity of other species of Trichinella, we discovered that a nearly uniform lineage of $T$. spiralis now occupies an exceptionally broad, trans-Atlantic distribution. More limited geographic ranges typify species of Trichinella restricted to wildlife hosts. The absence of regional differentiation between far-flung Western populations underscores the hypothesis that $T$. spiralis disseminated there only recently. Genealogies reconstructed from both the microsatellite and mitochondrial data indicate that the earliest diversifications in T. spiralis occurred in Asia. More intensive sampling in Asia might establish with greater precision where and when the Western lineage of $T$. spiralis originated. Stochastic sampling error renders molecular clock estimates imprecise even when rate calibrations are well-supported (Ayala, 1997; Hillis and Moritz, 1996), but extant Western isolates of $T$. spiralis share more recent common ancestry than do lineages of Trichinella evidently separated during the Pleistocene (i.e. T. nativa and the T6 genotype) (Zarlenga et al., 2006). Neither domesticated pigs nor their feral descendants inhabited the Americas before the Colonial Era. Our genetic data suggest that the same is true of $T$. spiralis.

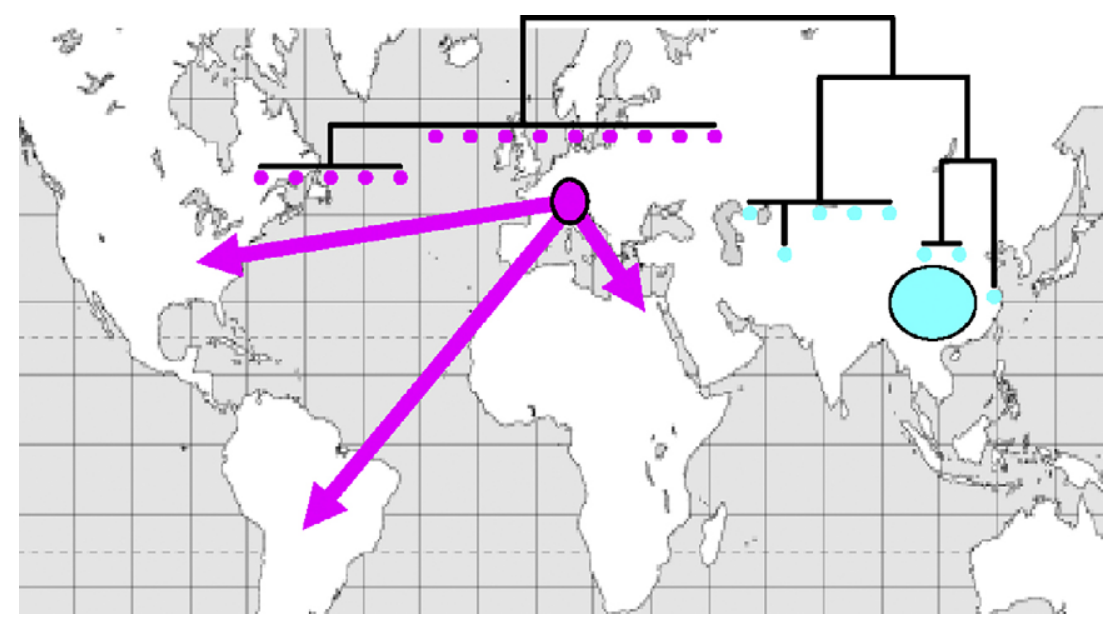

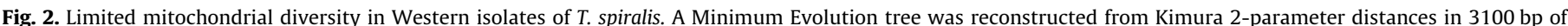

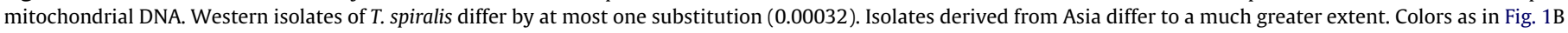
and $\mathrm{C}$. 
Human beings may have first consumed meats infected with Trichinella and other parasites millions of years before the advent of agriculture (Owen et al., 2005; Zarlenga et al., 2006). T. spiralis diverged early in the history of the genus; its distant relationship to other species of Trichinella therefore renders its ultimate origins enigmatic (Zarlenga et al., 2006). Evidence from other parasites ingested in meat suggest that Trichinella could have infected human beings millions of years before the domestication of pigs. For example, swine and cattle probably first became infected with tapeworms (of the genus Taenia) by Neolithic farmers, whose remote ancestors first became infected while consuming the traditional prey of hyenas (Hoberg et al., 2001). If such early hominid hunters were simultaneously exposed to infection with Trichinella, then the extant genetic variation in $T$. spiralis reflects only its most recent transmission and dispersal history.

The extant ecology and epidemiology of foodborne parasites has been profoundly influenced by livestock domestication. As may also be true for pork tapeworms (Campbell et al., 2006; Nakao et al., 2002), T. spiralis in the West originated in domesticated pigs only within the last several thousand years and was disseminated by European colonists only within the last several hundred years. Interestingly, the diversity in T. spiralis appears to be greater where pigs were first domesticated, in East Asia. There, the true diversity of $T$. spiralis undoubtedly exceeds that evident in the current, admittedly limited sample. By contrast, additional sampling of isolates in the West would seem unlikely to identify heretofore uncharacterized variants.

Although $T$. spiralis remains a parasite of animals, its abundance, distribution, and diversity were profoundly influenced by human activity. This distinguishes $T$. spiralis from other pathogens engendered by Eurasian agriculturalists and disseminated by their seafaring descendants (i.e. smallpox and measles viruses, which lost their dependency on animal reservoirs) and from other zoonoses requiring animal reservoirs (which, like anthrax and rabies, generally originated in the tropics prior to the advent of agriculture) (Wolfe et al., 2007). Thus, a distinct evolutionary ecology may characterize zoonotic pathogens of domesticated livestock.

The expansion of agriculture was evidently responsible for the historical dissemination of $T$. spiralis, but the requirements for ongoing transmission remain uncertain. In particular, it is unclear how extensive a risk wildlife or rats pose to swine where transmission among pigs has been prevented by eliminating, from their diet, meats harboring viable parasites. The species of Trichinella that most commonly infect wildlife pose negligible risk to the safety of pork (Kapel and Gamble, 2000). Nonetheless, the risks to swine posed by suspected wildlife reservoirs of $T$. spiralis should be evaluated in the various agro-ecological settings where swine are raised, because measures intended to safeguard food safety require substantial effort and cost. Does $T$. spiralis thrive primarily where poor management facilitates its transmission among swine (Kapel, 2000; Pozio, 2000; Pozio and Murrell, 2006), or has the parasite instead become established in selfsustaining cycles among wildlife (Rafter et al., 2005)? If eliminating $T$. spiralis from swine herds irrevocably breaks the cycle of transmission necessary for its local persistence, such interventions would provide enduring benefits to the economy and to public health.

\section{Acknowledgements}

We greatfully acknowledge financial support from USDA CRIS Project 701-1265-140-11 and the Organization for Economic Cooperation and Development's Co-Operative Research Pro- gramme for Biological Resource Management for Sustainable Agricultural Systems. All of those who deposited specimens with the International Trichinellosis Reference Center made this comparative study possible.

\section{References}

Arbogast, B.S., Edwards, S.V., Wakeley, J., Beerli, P., Slowinski, J.B., 2002. Estimating divergence times from molecular data on phylogenetic and population genetic timescales. Annu. Rev. Ecol. Syst. 33, 707-740.

Ayala, F.J., 1997. Vagaries of the molecular clock. Proc. Natl. Acad. Sci. U.S.A. 94, 7776-7783.

Brown, W.M., George, M., Wilson, A.C., 1979. Rapid evolution of animal mitochondrial DNA. Proc. Natl. Acad. Sci. U.S.A. 76, 1967-1971.

Campbell, G., Garcia, H.H., Nakao, M., Ito, A., Craig, P.S., 2006. Genetic variation in Taenia solium. Parasitol. Int. 55 (Suppl.), S121-126.

Clutton-Brock, J., 1999. A Natural History of Domesticated Mammals Cambridge. Cambridge University Press.

Crosby, A.W., 1976. Virgin soil epidemics as a factor in the aboriginal depopulation in America. William Mary Q 33, 289-299.

Crosby, A.W., 1986. Ecological Imperialism: The Biological Expansion of Europe 900-1900. Cambridge University Press, Cambridge.

Dame, J.B., Murrell, K.D., Worley, D.E., Schad, G.A., 1987. Trichinella spiralis: Genetic evidence for synanthropic subspecies in sylvatic hosts. Exp. Parasitol. 64, 195203.

Diamond, J., 2002. Evolution, consequences and future of plant and animal domestication. Nature 418, 700-707.

Dupouy-Camet, J., Kociecka, W., Bruschi, F., Bolas-Fernandez, F., Pozio, E., 2002. Opinion on the diagnosis and treatment of human trichinellosis. Expert. Opin. Pharmacother. 3, 1117-1130.

Gamble, H.R., Murrell, K.D., 1986. Conservation of diagnostic antigen epitopes among biologically diverse isolates of Trichinella spiralis. J. Parasitol. 72, 921925.

Goldstein, D.B., Ruiz Linares, A., Cavalli-Sforza, L.L., Feldman, M.W., 1995. Genetic absolute dating based on microsatellites and the origin of modern humans. Proc. Natl. Acad. Sci. U.S.A. 92, 6723-6727.

Hillis, D.M.B.K.M., Moritz, C., 1996. Applications of molecular systematics: the state of the field and a look to the future. In: Hillis, D.M.C.M., Mable, B.K. (Eds.), Molecular Systematics. 2nd ed. Sinauer Associates, Sunderland, MA, pp. 531-540.

Hoberg, E.P., Alkire, N.L., de Queiroz, A., Jones, A., 2001. Out of Africa: origins of the Taenia tapeworms in humans. Proc. Biol. Sci. 268, 781-787.

Horimoto, T., Kawaoka, Y., 2005. Influenza: lessons from past pandemics, warnings from current incidents. Nat. Rev. Microbiol. 3, 591-600.

Kapel, C.M., 2000. Host diversity and biological characteristics of the Trichinella genotypes and their effect on transmission. Vet. Parasitol. 93, 263-278.

Kapel, C.M., Gamble, H.R., 2000. Infectivity, persistence, and antibody response to domestic and sylvatic Trichinella spp. in experimentally infected pigs. Int. J. Parasitol. 30, 215-221.

Larson, G., Albarella, U., Dobney, K., Rowley-Conwy, P., Schibler, J., Tresset, A., Vigne, J.D., Edwards, C.J., Schlumbaum, A., Dinu, A., Balacsescu, A., Dolman, G., Tagliacozzo, A., Manaseryan, N., Miracle, P., Van Wijngaarden-Bakker, L., Masseti, M., Bradley, D.G., Cooper, A., 2007. Ancient DNA, pig domestication, and the spread of the Neolithic into Europe. Proc. Natl. Acad. Sci. U.S.A. 104, 15276-15281.

Larson, G., Dobney, K., Albarella, U., Fang, M., Matisoo-Smith, E., Robins, J., Lowden, S., Finlayson, H., Brand, T., Willerslev, E., Rowley-Conwy, P., Andersson, L., Cooper, A., 2005. Worldwide phylogeography of wild boar reveals multiple centers of pig domestication. Science 307, 1618-1621.

Lavrov, D.V., Brown, W.M., 2001. Trichinella spiralis mtDNA: a nematode mitochondrial genome that encodes a putative ATP8 and normally structured tRNAs and has a gene arrangement relatable to those of Coelomate metazoans. Genetics 157, 621-637.

Mason, I.L., 1986. Evolution of Domesticated Animals. John Wiley \& Sons.

Murrell, K., Leiby, D.A., Duffy, C.H., Schad, G.A. (Eds.), 1985. Susceptibility of Domestic Swine to Wild Animal Isolates of Trichinella spiralis. The State University of New York Press, Albany.

Nakao, M., Okamoto, M., Sako, Y., Yamasaki, H., Nakaya, K., Ito, A., 2002. A phylogenetic hypothesis for the distribution of two genotypes of the pig tapeworm Taenia solium worldwide. Parasitology 124, 657-662.

Owen, I.L., Gomez Morales, M.A., Pezzotti, P., Pozio, E., 2005. Trichinella infection in a hunting population of Papua New Guinea suggests an ancient relationship between Trichinella and human beings. Trans. R. Soc. Trop. Med. Hyg. 99, 618 624.

Palumbi, S.R., 2001. Humans as the world's greatest evolutionary force. Science 293, 1786-1790.

Pozio, E., 2000. Factors affecting the flow among domestic, synanthropic and sylvatic cycles of Trichinella. Vet. Parasitol. 93, 241-262.

Pozio, E., Murrell, K.D., 2006. Systematics and epidemiology of Trichinella. Adv. Parasitol. 63, 367-439.

Pritchard, J.K., Stephens, M., Donnelly, P., 2000. Inference of population structure using multilocus genotype data. Genetics 155, 945-959.

Rafter, P., Marucci, G., Brangan, P., Pozio, E., 2005. Rediscovery of Trichinella spiralis in red foxes (Vulpes vulpes) in Ireland after 30 years of oblivion. J. Infect. 50, 61-65. 
Shriver, M.D., Jin, L., Boerwinkle, E., Deka, R., Ferrell, R.E., Chakraborty, R., 1995. A novel measure of genetic distance for highly polymorphic tandem repeat loci. Mol. Biol. Evol. 12, 914-920.

Tamura, K., Dudley, J., Nei, M., Kumar, S., 2007. MEGA4: Molecular Evolutionary Genetics Analysis (MEGA) software version 4.0. Mol. Biol. Evol. 24, 1596.

Tibayrenc, M., 2001. The relevance of evolutionary genetics for identification of Trichinella sp. and other pathogens at the strain, subspecies and species levels. Parasite 8, S21-23.
Wolfe, N.D., Dunavan, C.P., Diamond, J., 2007. Origins of major human infectious diseases. Nature 447, 279-283.

Zarlenga, D.S., Barta, J.R., 1990. DNA analysis in the diagnosis of infection and in the speciation of nematode parasites. Rev. Sci. Technol. 9, 533-554.

Zarlenga, D.S., Rosenthal, B.M., La Rosa, G., Pozio, E., Hoberg, E.P., 2006. PostMiocene expansion, colonization, and host switching drove speciation among extant nematodes of the archaic genus Trichinella. Proc. Natl. Acad. Sci. U.S.A. 103, 7354-7359. 